\title{
Flexible graphene-based supercapacitors: a review
}

\begin{abstract}
The recent rapid growth in graphene-based supercapacitors has reached the point where there is a need for solid-state devices with physical flexibility, which will be a crucial advantage in modern electronic devices. Herein, we summarize recent developments toward an all solidstate graphene-based flexible supercapacitor. The routes to produce graphene-based electrode materials, along with the typical fabrication techniques for flexible devices, are thoroughly discussed. Furthermore, the structural morphology of the electrode materials is closely related to the electrochemical performance, and the influence of the electrode components on the mechanical flexibility of the fabricated devices is examined. Lastly, a summary of the overall electrochemical properties and current development of the reported devices is presented progressively to predict the future trends toward the realization of an ultimate-performance graphene-based flexible supercapacitor.
\end{abstract}

Keyword: Flexible graphene-based supercapacitors; Electronic device 